\title{
Association Between a Visual and an Automated Locomotion Score in Lactating Holstein Cows
}

\author{
R. C. Bicalho, ${ }^{\star 1}$ S. H. Cheong, ${ }^{*}$ G. Cramer, $\dagger$ and C. L. Guard ${ }^{\star}$ \\ *Department of Population Medicine and Diagnostic Sciences, College of Veterinary Medicine, Cornell University, Ithaca, NY 14853 \\ †Department of Population Medicine, Ontario Veterinary College, University of Guelph, Ontario, Canada
}

\begin{abstract}
Two studies were conducted to evaluate visual locomotion scoring (VLS) and Stepmetrix locomotion scoring (SLS) in detecting painful digit lesions. In study 1 , one veterinarian performed VLS. Cows with VLS $\geq 3$ were hoof trimmed and the presence or absence of a painful lesion (PL), defined as a reaction to digital pressure, was recorded. A strongly increasing pattern in the proportion of cows with PL was detected as VLS increased. The proportions of cows with painful lesions were $5.6 \%(\mathrm{n}=53), 20.1 \%(\mathrm{n}=78), 55.5 \%(\mathrm{n}=164)$, $79.9 \%(\mathrm{n}=159)$, and $100 \%(\mathrm{n}=5)$ for VLS 1 to 5 , respectively. Study 2 was conducted on a different farm. The entire farm was visually locomotion scored by 3 veterinarians on the same day, and the cows were Stepmetrix locomotion scored by walking through the Stepmetrix system. Every cow was trimmed during the following $2 \mathrm{~d}$ by 1 of 8 professional hoof trimmers. The 3 veterinarians identified, scored, and recorded any PL. Interobserver agreement for the 3 veterinarians had a kappa coefficient of between 0.45 and $0.48 \pm 0.05$. In total, 518 cows were used in the analysis, from which $11.2 \%$ were identified with a PL. Of the cows diagnosed with a PL, $32.8 \%$ were detected with a sole ulcer, $25.9 \%$ with white line disease, $13.8 \%$ with white line abscess, and $27.5 \%$ with other diseases. A receiver operating characteristic analysis was performed; the area under the curve was larger for VLS $(0.80 ; 95 \%$ confidence interval, 0.76 to 0.83$)$ than SLS $(0.62 ; 95 \%$ confidence interval, 0.57 to 0.66 ). When performed by trained veterinarians, VLS performed better than SLS in detecting PL.
\end{abstract}

Key words: lameness, Stepmetrix, dairy cow

\section{INTRODUCTION}

Lameness is a prevalent and costly disease in modern dairy production (Murray et al., 1996; Green et al.,

Received February 5, 2007.

Accepted March 26, 2007.

${ }^{1}$ Corresponding author: rcb28@cornell.edu
2002). It is perhaps the greatest challenge for dairies to overcome, as the public have become more concerned about the origins of their food and the welfare of farm animals. Furthermore, the dairy industry today lacks a documented method, with proven accuracy, for identifying lame cows with lesions amenable to therapy.

Claw disorders account for approximately $90 \%$ of lameness in dairy cattle (Murray et al., 1996). Early detection and treatment of lameness reduces economic losses and the prevalence of lameness (Clarkson et al., 1996; Espejo et al., 2006). Lameness is usually detected by visual observation of abnormal locomotion by the farmer or farm personnel. As farms grow larger, the ratio of cows to farm personnel increases, and this reduces the likelihood that a lame cow will be detected and treated in a timely fashion. This has led to interest in automated methods of lameness detection (Neveux et al., 2006). Whay et al. (2003) reported that dairy producers are aware of only 25 to $50 \%$ of the cows that are lame, and Wells et al. (1993) reported that producers correctly identified only 40 to $45 \%$ of lame cows. Scoring herds for locomotion is important for observing the prevalence of lameness in the herd and detecting cows that require treatment.

Biomechanical technology for lameness detection in cattle has been researched, such as the video motion analysis by Flower et al. (2005) and ground reaction force detection by Rajkondawar et al. (2006). Although studies have shown encouraging results, the use of automated lameness detection is not widely used. One automated lameness detection system, Stepmetrix (BouMatic, Madison, WI), which uses ground reaction force measurements, is commercially available.

Several visual locomotion scoring (VLS) systems have been described (Sprecher et al., 1997; Engel et al., 2003); however, the lack of a gold standard for the presence of lameness makes it difficult to evaluate the accuracy and optimal cutoff point for intervention. The presence of a painful lesion in the foot, such as a sole ulcer or an abscess, which is diagnosed at hoof trimming, should be associated with an abnormal gait.

The objectives of this study were to evaluate the association between VLS and an automated locomotion scor- 
ing system (Stepmetrix locomotion scoring, SLS) in the presence or absence of painful digit lesions to compare the accuracy of VLS vs. SLS for detecting painful foot lesions. Our hypotheses were that a significant association would be found between VLS or SLS and painful foot lesions, and that VLS would have a higher accuracy in detecting painful foot lesions than SLS.

\section{MATERIALS AND METHODS}

\section{Study 1}

Farm and Management. Data were collected from one dairy farm located near Ithaca, New York, from October 15, 2005, to April 15, 2006. The farm milked 2,800 Holstein cows 3 times daily in a double-54 milking parlor. The cows were housed in free-stall barns with concrete stalls covered with mattresses and bedded with wastepaper pulp. Feed alleys were grooved concrete flooring and cleaned by automatic scrapers. All walkways leading to and from the milking barn and the holding pen were covered with rubber flooring. Footbaths were located in the exit lanes of the milking parlor. The footbath consisted of a $5 \%$ formalin solution and was applied a minimum of 4 times weekly. Every cow was scheduled to receive routine hoof trimming twice yearly. Lame cows were identified and treated by trained farm employees. Moreover, cows enrolled in this study were not trimmed by the farm employees.

Design and Data Collection. A prospective observational study design was used. Three pens of lactating dairy cows, 1 pen with 310 cows and 2 pens with 150 cows each, were enrolled in the study. The criteria for selection of the pens were based on pen location, farm milking schedule, and ability of the veterinarian present to score cows at a fixed time every week. Enrolled cows were visual locomotion scored as the cows exited the milking parlor. Scoring was done by the same veterinarian and the system used was a 5-point scoring scale, where $1=$ normal, $2=$ presence of a slightly asymmetric gait, $3=$ cow clearly favors one or more limbs (moderately lame), $4=$ severely lame, or $5=$ extremely lame (non-weight-bearing lame). The veterinarian recorded the cow identification number, VLS, and suspected affected limb for VLS $\geq 3$ into a digital voice recorder while the cows were walking by. There were 7 categories of affected limbs by visual assessment: left front, left hind, right front, right hind, both hind, both front, and all limbs. On the same day, the data were entered into a database (Access, Microsoft Corporation, Redmond, WA) created for the study.

A parallel clinical study was done to evaluate the effect of treatment on lame cows using 2 different VLS thresholds. At the beginning of the study, every cow on the farm was randomly assigned a code of 1 or 2 . Code
1 cows would be considered lame on a VLS of 4 or 5 (LVLS4), and code 2 cows were considered lame if VLS was 3 , 4 , or 5 (LVLS3). Therefore, only a portion of the cows with a VLS of 3 were treated.

Criteria for treatment were a VLS of 3 (for LVLS3 cows), 4 , or 5 , and cows scheduled for routine hoof trimming. In total, 246 cows were allocated to trimming because they were detected with a VLS $\geq 3$, and 213 cows were allocated to trimming because they were due for a routine trim. Selected cows were trimmed the day after VLS or the following day if too many cows were scheduled for treatment. The treatment lists were generated at the end of data entry each week using the study database in Microsoft Access according to historical treatment data and VLS. Treated cows would not return to the trimming table for the following $2 \mathrm{wk}$ unless an increase in locomotion score was observed for that period. All hoof trimmings were done by the same veterinarian. Cows were trimmed in a standing hooftrimming chute and all 4 feet were trimmed and examined. Lesions were treated, recorded, and graded according to severity. A list of 15 diseases was created to categorize the lesions. Cows were classified as having either the presence or absence of a painful lesion (PL). Pain was defined by reaction to gentle pressure applied to the lesions by hand. In the instance of a clearly PL, such as a sole ulcer with substantial exposure of corium tissue, the cow was spared from having pressure applied to the lesion but was classified as having a PL. All PL were scored for severity. Severity 1 was used for mild lesions and severity 2 was used to classify advanced lesions. Affected limb or limbs identified at trimming were recorded using the same categories as for the visual assessment of affected limbs.

Only the first treatment of each cow was used for analyses. Although data were collected after the first treatment, no data for that cow were used after the first treatment date.

Statistical Analysis. For study 1, accuracy analyses (sensitivity, specificity, positive predictive value, and negative predictive value) were not performed, because the research sample was not representative of the farm population; animals assigned for hoof trimming were not randomly selected from the population.

An increasing or decreasing trend on the frequency of PL by VLS, and disease severity by VLS was evaluated using the Cochran-Armitage trend test using PROC FREQ of SAS (SAS Institute, Cary, NC).

The variable VLS was dichotomized twice, which generated 2 new variables, LVLS3 and LVLS4. For LVLS3, the cows classified as 1 or 2 at VLS were considered sound and were therefore classified as LVLS3 $=0$; the cows classified from 3 to 5 at VLS were considered lame and were therefore classified as LVLS3 $=1$. For LVLS4, 
the cows classified as 1 to 3 at VLS were considered sound and were therefore classified as LVLS $4=0$; the cows classified as 4 or 5 at VLS were considered lame and were therefore classified as LVLS4 $=1$. To analyze the association between VLS and the presence or absence of a PL, a Spearman correlation coefficient was calculated using PROC FREQ of SAS, with the PLCORR option.

To facilitate analysis and interpretation of the association between affected limb at visual scoring and affected limb at treatment, the variables were dichotomized into front limb and hind limb lameness. The association between affected limb at visual scoring and affected limb at treatment was analyzed using the Spearman correlation coefficient.

\section{Study 2}

Farm and Management. Data were collected from one dairy farm located in Lancaster County, Pennsylvania, from March 15 to 18, 2006. The farm milked 600 Holstein cows 3 times daily in a double-12 milking parlor. Lactating cows were fed a TMR. The cows were housed in free-stall barns with slatted floors. The concrete stalls were covered with mattresses, and the tops of the mattresses were bedded daily with wood shavings. Footbaths were located in the exit lanes of the milking parlor. Every cow was scheduled to receive routine hoof trimming at least twice yearly. Lame cows were identified and treated by trained farm employees and professional hoof trimmers. Moreover, cows enrolled in this study were not trimmed by the farm employees.

Design and Data Collection. A prospective observational study design was used. All cows were scored using VLS once as they were exiting the milking parlor. Scoring was done by 3 veterinarians who were previously trained to perform VLS. The VLS system used was identical to that used in study 1 . The veterinarians recorded the cow identification number, VLS, and suspected affected limb for VLS $\geq 3$ into digital voice recorders while the cows were walking by. There were 7 categories of affected limb, as in study 1 . The data were entered into a database (Microsoft Access) created for the study on the same day.

The Stepmetrix machine was located in a single-lane alley returning to the free-stall barn. The research cows walked through the Stepmetrix machine 3 times daily when returning from the milking parlor. A report, consisting of an SLS for each lactating cow, was generated by the Stepmetrix software and recorded on the same day the VLS were performed. The SLS was an average of all measurements captured for each cow for a period of $7 \mathrm{~d}$. The SLS ranged from 1 to 100 . The manufactur- er's suggested cutoff point for SLS was 39; cows with SLS of $\geq 39$ were considered lame.

For the $2 \mathrm{~d}$ following the day of VLS, 8 professional hoof trimmers working as a group and using 4 professional hoof-trimming chutes performed maintenance hoof trimming and, whenever necessary, therapeutic trimming on all lactating cows. The same group of veterinarians that performed the VLS inspected the cows' feet while the cows were being trimmed; diseases were recorded based on the same list of diseases previously described for study 1 . Cows were classified as having either the presence or absence of a PL.

Statistical Analysis. Three veterinarians performed VLS. Interobserver agreement for the VLS performed by the different veterinarians was assessed using the weighted kappa (K) statistic (Cohen's kappa), calculated using MedCalc (www.medcalc.be). Furthermore, a receiver operating characteristic (ROC) analysis was performed for each veterinarian, assuming detection of PL at hoof trimming as the reference test. For the accuracy analysis, the mean of the available scores for the 3 veterinarians was calculated and rounded to a decimal point. If a cow had been scored by only 2 veterinarians, but not by the third veterinarian, the mean was calculated by adding the 2 scores and dividing by 2 .

For study 2 , sensitivity, specificity, positive predictive value, and negative predictive value analyses were conducted, assuming that the diagnosis of PL during hoof trimming was the gold standard method (reference test) for detection of painful digit lesions. This accuracy analysis was done using MedCalc. Differences between SLS and VLS in the accuracy of detecting PL were evaluated by ROC curves, by estimating differences of the area under the ROC curves and by using the statistical software package MedCalc.

\section{RESULTS}

\section{Study 1}

Frequency of Disease. A total of 459 cows were evaluated by hoof trimming, of which $52.7 \%$ were classified as having a PL. Sole ulcer was the most common disease, accounting for $52.1 \%(\mathrm{n}=242)$ of all PL. In descending order, the rest of the observed digit diseases were digital dermatitis (19.8\%), white line abscess (14.5\%), toe ulcer (2.9\%), heel ulcer (2.6\%), double sole $(2.3 \%)$, foot rot (1.0\%), and other diseases $(4.8 \%)$.

Presence of a PL by VLS. A total of 289 cows had $\mathrm{PL}$ at first trimming. An increasing pattern in the proportion of cows with PL was detected as the VLS increased $(P<0.001$; Figure 1). The proportions of cows with PL were 5.6, 20.1, 55.5, 79.9, and $100 \%$ for VLS 1 to 5 , respectively. 


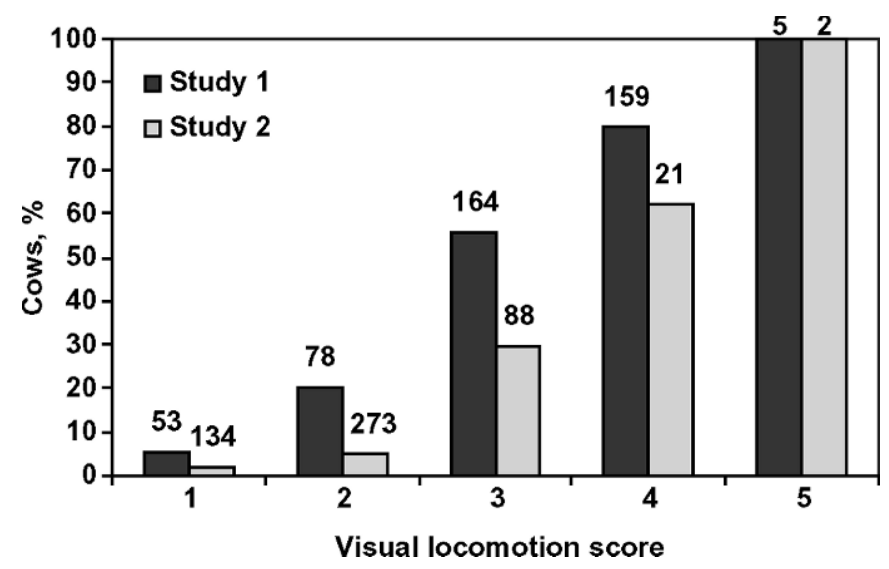

Figure 1. Proportion of cows with painful lesions (PL) detected at hoof trimming by visual locomotion score (VLS). Black bars represent cows from study 1 and gray bars represent the cows from study 2 . For both studies, the increasing trend in the proportion of PL as the VLS increased was significant with the Cochran-Armitage trend test. The numbers above the bars represent the total number of cows per category.

The VLS were dichotomized twice, first considering VLS $\geq 3$ as being lame and then considering VLS $\geq 4$ as being lame, and a correlation analysis was done to measure the degree of association of the 2 dichotomized VLS and the presence or absence of a PL. When cows with VLS $\geq 3$ were considered lame and the cows with VLS $<3$ were considered sound, the Spearman correlation coefficient was $0.48 \pm 0.04$. When cows were considered lame only if their VLS were $\geq 4$ and cows with VLS $<4$ were considered sound, the Spearman coefficient was $0.41 \pm 0.04$ (Table 1).

Association of Affected Limb. A total of 215 cows that were lame by VLS and had PL on hoof trimming were included in this analysis. Overall, the association between affected limb at VLS and at hoof trimming was meager; of all the cows diagnosed as having front limb lameness at VLS, $54.4 \%(\mathrm{n}=57)$ actually had a lesion in the front digit detected at hoof trimming. For all cows diagnosed as having lameness in one or both hind feet at VLS, $76.0 \%(\mathrm{n}=158)$ actually had a PL diagnosed during hoof trimming. The Spearman correlation coefficient was $0.29 \pm 0.07$ (Table 2).

\section{Study 2}

Frequency of Disease. In total, 528 cows were evaluated by hoof trimming, of which $12.9 \%$ were classified as having a PL. White line abscess was the most common disease, accounting for $38.2 \%$ of all PL. In descending order, the rest of the observed digit diseases were sole ulcers (30.9\%), digital dermatitis (8.9\%), heel ulcer $(7.4 \%)$, toe ulcer $(4.4 \%)$, and other diseases $(10.3 \%)$.

Interobserver VLS Agreement. Agreement beyond chance was performed among the observers that performed VLS, according to the kappa statistic analysis. The kappa coefficient for observers 1 and 2 was $0.46 \pm$ $0.05(\mathrm{n}=459)$. A complete summary of the kappa statistic for all comparisons is shown in Table 3.

An ROC analysis was performed to compare the area under the curve for each veterinarian that performed VLS (Figure 2). The area under the curve was 0.77 [confidence interval $(\mathbf{C I})=0.72$ to 0.81 ], $0.74(\mathrm{CI}=0.69$ to 0.78 ), and $0.76(\mathrm{CI}=0.71$ to 0.80 ) for veterinarians 1,2 , and 3, respectively].

Presence of a PL by VLS. The VLS used in the analysis was the mean of 3 locomotion scores taken by the different observers; the numbers were rounded to the nearest digit so that the end numbers would fall back into a 5-point scale categorical variable.

After excluding the cows with missing data, a total of 518 cows had complete data and were used for the final analysis. The overall prevalence of PL was $11.2 \%$

Table 1. Association analysis comparing visual locomotion scores (VLS) at 2 different thresholds: lame if VLS $\geq 3$ or lame if VLS $\geq 4^{1}$

\begin{tabular}{|c|c|c|}
\hline \multirow[b]{2}{*}{ Score } & \multicolumn{2}{|c|}{ Cutoff of 3} \\
\hline & No PL, \% & PL, \% \\
\hline VLS $<3$ & 24.40 & 4.14 \\
\hline VLS $\geq 3$ & 22.88 & 48.58 \\
\hline \multirow[t]{2}{*}{ Spearman correlation coefficient (adjusted SE) } & \multicolumn{2}{|c|}{$0.48(0.04)$} \\
\hline & \multicolumn{2}{|c|}{ Cutoff of 4} \\
\hline Score & No PL, \% & PL, \% \\
\hline VLS $<4$ & 40.31 & 23.97 \\
\hline VLS $\geq 4$ & 6.97 & 28.76 \\
\hline Spearman correlation coefficient (adjusted SE) & \multicolumn{2}{|c|}{$0.41(0.04)$} \\
\hline
\end{tabular}

\footnotetext{
${ }^{1}$ The reference test used was the presence or absence of a painful lesion (PL or No PL) detected at hoof trimming. Spearman correlation coefficients and their respective SE are provided for study 1. Total number of observations $=459$.
} 
Table 2. Correlation table of an affected limb at visual locomotion scoring (VLS) and an affected limb at treatment, with the Spearman correlation coefficient in study 1

\begin{tabular}{|c|c|c|}
\hline Limb & $\begin{array}{c}\text { Front at } \\
\text { treatment, }{ }^{1} \%\end{array}$ & $\begin{array}{c}\text { Hind at } \\
\text { treatment, }{ }^{2} \%\end{array}$ \\
\hline Front at $\operatorname{VLS}^{3}(n=57)$ & 54.4 & 45.6 \\
\hline Hind at $\operatorname{VLS}^{4}(n=158)$ & 24.0 & 76.0 \\
\hline Spearman correlation coefficient (adjusted SE) & \multicolumn{2}{|c|}{$0.29(0.07)$} \\
\hline
\end{tabular}

${ }^{1}$ Front at treatment = a painful lesion was diagnosed in one or both front limbs during hoof trimming.

${ }^{2}$ Hind at treatment $=$ a painful lesion was diagnosed in one or both hind limbs during hoof trimming.

${ }^{3}$ Front at VLS $=$ observer suspects one or both front limbs with lesions are causing lameness.

${ }^{4}$ Hind at VLS = observer suspects one or both hind limbs with lesions are causing lameness.

$(\mathrm{n}=518)$. The most prevalent disease was sole ulcer $(32.8 \%, \mathrm{n}=58)$, followed by white line disease $(25.9 \%$, $\mathrm{n}=58)$, white line abscesses $(13.8 \%, \mathrm{n}=58)$, digital dermatitis $(8.7 \%, \mathrm{n}=58)$, and others $(18.8 \%, \mathrm{n}=58)$. A strongly increasing pattern in the proportion of cows with PL was detected as VLS increased $(P<0.001)$. The proportions of cows with PL were 2.2, 5.1, 29.6, 61.9 , and $100 \%$ for VLS of 1 to 5 , respectively (Figure 1).

Accuracy Analyses for VLS by a PL. An accuracy analysis for VLS by PL was performed using the ROC curve. When cows classified as VLS 3, 4, and 5 were considered lame, the sensitivity of VLS to detect PL was $67.5 \%$ (95\% CI, 54.6 to $78.1 \%$ ). The specificity for this same cutoff was $84.6 \%$ (95\% CI, 81.0 to $87.8 \%$ ). A complete summary of the accuracy analysis for VLS is provided in Table 4.

Accuracy Analyses for SLS by a PL. Accuracy was assessed for SLS by a PL. When the cows with an SLS score greater than 39 were considered lame, the sensitivity of SLS in detecting a PL was $22.2 \%$ (95\% CI, 12.1 to $35.6 \%)$. The specificity for this same cutoff was $93.8 \%$ (95\% CI, 91.2 to $95.9 \%$ ). A complete summary of the accuracy analysis for SLS is provided in Table 5.

ROC Comparisons for VLS and SLS by a PL. A comparison of the ROC curves for VLS and SLS was performed with the hypothesis that the areas under

Table 3. Analysis of agreement among visual locomotion score observ$\operatorname{ers}^{1}$

\begin{tabular}{lccc}
\hline Item $^{2}$ & $\mathrm{~K}$ & $\mathrm{SE}^{3}$ & $\mathrm{n}^{4}$ \\
\hline Obs1 Obs2 & 0.46 & 0.05 & 459 \\
Obs1 Obs3 & 0.45 & 0.05 & 402 \\
Obs2 Obs3 & 0.48 & 0.05 & 424 \\
\hline
\end{tabular}

${ }^{1}$ Kappa coefficients $(\mathrm{K})$ and respective SE are presented for each comparison in study 2.

${ }^{2}$ Obs 1 Obs2 = agreement between visual locomotion scores by observer 1 and observer 2; Obs1 Obs3 = agreement between visual locomotion scores by observer 1 and observer 3; Obs2 Obs3 = agreement between visual locomotion scores by observer 2 and observer 3.

${ }^{3} \mathrm{SE}=\mathrm{SE}$ of kappa coefficient.

${ }^{4} \mathrm{n}=$ total number of scores used for each analysis. the different curves were significantly different. A total of 492 cows were used in this analysis. For the VLS, the area under the ROC curve was 0.77 (95\% CI, 0.74 to $0.81 \%$ ) and for the SLS the area under the ROC curve was 0.62 (95\% CI, 0.57 to $0.66 \%$; Figure 3 ).

\section{DISCUSSION}

The 2 studies were conducted concurrently by the same researchers. The VLS system and PL scoring used for both studies were the same. The definition of a PL used in both studies was reaction when digital pressure was applied to the lesion or if the cow had an obviously PL, such as a sole ulcer with significant corium exposure or a white line abscess with great separation be-

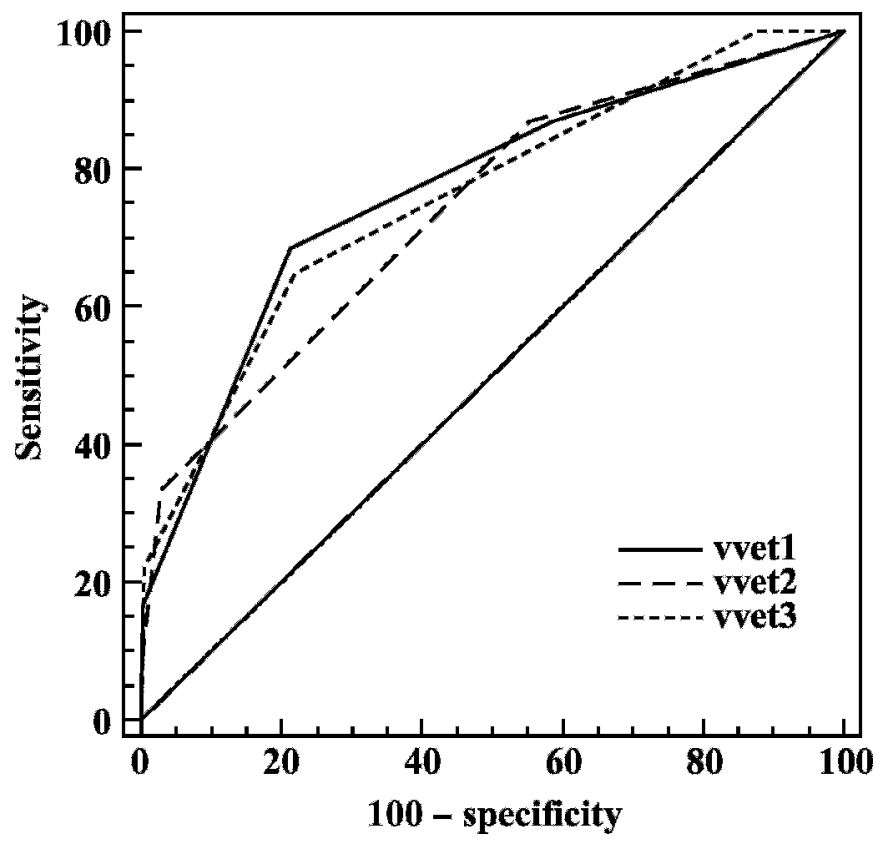

Figure 2. Receiver operating characteristic curve of visual locomotion scores for veterinarians 1,2 , and 3 (vvet1, vvet2, and vvet3) by the presence or absence of a painful lesion detected at hoof trimming. There was no difference in the areas under the curves. This graph illustrates the results from study 2 . 
Table 4. Outcomes of the receiver operating characteristic curve analysis for visual locomotion scoring (VLS) by presence or absence of a painful lesion ${ }^{1}$

\begin{tabular}{lcccc}
\hline Criterion $^{2}$ & $\begin{array}{c}\text { Sensitivity, } \\
\%\end{array}$ & $\begin{array}{c}95 \% \text { CI, } \\
\%\end{array}$ & $\begin{array}{c}\text { Specificity, } \\
\%\end{array}$ & $\begin{array}{c}95 \% \text { CI, } \\
\%\end{array}$ \\
\hline VLS $>1$ & 94.03 & $85.4-98.3$ & 28.42 & $24.3-32.8$ \\
VLS $>2$ & 67.16 & $54.6-78.1$ & 84.60 & $81.0-87.8$ \\
VLS $>3$ & 23.88 & $14.3-35.9$ & 98.05 & $96.3-99.1$ \\
VLS $>4$ & 1.49 & $0.2-8.1$ & 99.78 & $98.8-100.0$ \\
\hline
\end{tabular}

${ }^{1}$ Sensitivity and specificity values and their respective confidence intervals (CI) by the different cutoff points of VLS for study 2 .

${ }^{2}$ Criterion = cutoff points used to dichotomize the VLS into lame or not lame. VLS $>1$ = considered lame if the VLS was 2 or greater; VLS $>2=$ considered lame if the VLS was 3 or greater; VLS $>3=$ considered lame if the VLS was 4 or greater; VLS $>4=$ considered lame if the VLS was 5 .

tween the corium tissue and the hoof wall or sole. The subjectivity of the definition of painful digit lesions is a disadvantage that the authors would like to acknowledge.

The frequency of disease diagnosed in study 1 represents the disease that caused the first episode of lameness during the study period, whereas the frequency of disease in study 2 represents the prevalence of disease at the time of whole-herd trimming. In study 1, the cows that had VLS of 1 and 2 but were trimmed were the cows scheduled for routine hoof trimming. These cows would not have been trimmed for 6 mo or more and were therefore at a higher risk of hoof overgrowth and infectious claw diseases (Fjeldaas et al., 2006) than if they had been chosen at random within all the cows with VLS of 1 or 2 . This might have caused a bias by increasing the proportion of PL detected in cows with VLS of 1 and 2 .

Table 5. Outcomes of the receiver operating characteristic curve analysis for Stepmetrix locomotion scores (SLS) by the presence or absence of a painful lesion ${ }^{1}$

\begin{tabular}{lcccc}
\hline & $\begin{array}{c}\text { Sensitivity, } \\
\%\end{array}$ & $\begin{array}{c}95 \% \text { CI, } \\
\text { Criterion }\end{array}$ & $\begin{array}{c}\text { Specificity, } \\
\%\end{array}$ & $\begin{array}{c}95 \% \text { CI, } \\
\%\end{array}$ \\
\hline SLS $>31$ & 35.19 & $22.7-49.4$ & 85.39 & $81.7-88.6$ \\
SLS $>32$ & 33.33 & $21.1-47.5$ & 87.21 & $83.7-90.2$ \\
SLS $>33^{3}$ & 33.33 & $21.1-47.5$ & 89.50 & $86.2-92.2$ \\
SLS $>34$ & 27.78 & $16.5-41.6$ & 90.41 & $87.3-93.0$ \\
SLS $>35$ & 24.07 & $13.5-37.6$ & 91.55 & $88.5-94.0$ \\
SLS $>36$ & 24.07 & $13.5-37.6$ & 92.24 & $89.3-94.6$ \\
SLS $>37$ & 24.07 & $13.5-37.6$ & 92.92 & $90.1-95.1$ \\
SLS $>38$ & 24.07 & $13.5-37.6$ & 93.61 & $90.9-95.7$ \\
SLS $>39$ & 22.22 & $12.1-35.6$ & 93.84 & $91.2-95.9$ \\
SLS $>40$ & 20.37 & $10.6-33.5$ & 94.52 & $92.0-96.5$ \\
\hline
\end{tabular}

${ }^{1}$ Sensitivity and specificity values and their respective confidence intervals (CI) by the different cutoff points of SLS in study 2 . Stepmetrix system (BouMatic, Madison, WI).

${ }^{2}$ Criterion = cutoff point used to dichotomize the SLS into lame or not lame. ship.

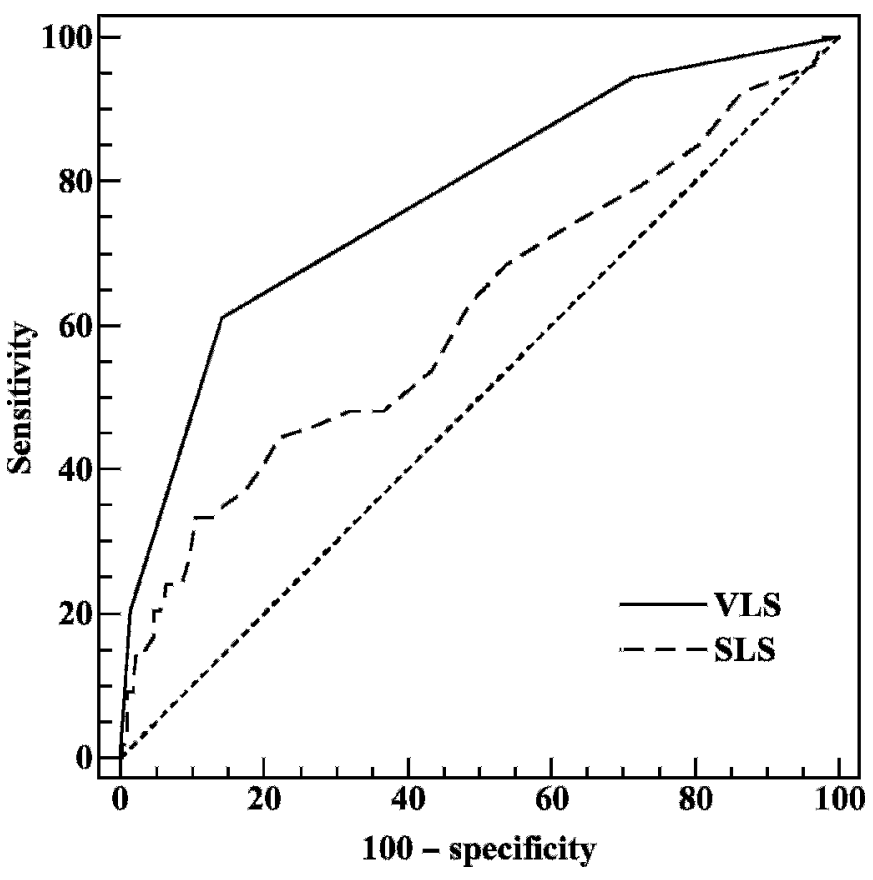

Figure 3. Receiver operating characteristic curve of Stepmetrix (BouMatic, Madison, WI) locomotion scores (SLS, dashed line) and visual locomotion scores (VLS, solid line) by the presence or absence of a painful lesion detected at hoof trimming. This graph illustrates the results from study 2 . The area under the curve was larger for VLS $(0.77 ; 95 \%$ CI, 0.74 to $0.81 \%)$ than for SLS $(0.62 ; 95 \%$ CI 0.57 to $0.66 \%$ ).

There are many limitations to visual observations as a method for diagnosing lameness, such as the subjective nature of locomotion scoring systems (Neveux et al., 2006), the reliance on the skill of the observer to detect gait abnormalities (Flower et al., 2005), and the variation between and within observers (O'Callaghan et al., 2003). Despite these shortcomings, only $15.5 \%$ of cows with VLS of 1 or 2 in study 1 had a PL, $80.0 \%$ of cows with VLS of 4 or 5 had a PL, and $54.9 \%$ of cows with VLS of 3 had a PL. Visual locomotion score 3 represented $36.7 \%$ of all trimmings. Hoof trimming is time-consuming and expensive; therefore, the benefits of trimming cows with VLS of 3 needs to be studied further to determine if intervention in these cows is warranted.

In study 1 , only one person was performing locomotion scoring; therefore, analyses of interobserver agreement were not possible. Cows in study 1 were visual locomotion scored every week, and some cows might not have had time for the lesions to develop into PL detectable by trimming. Cows were scored every week, and only the first trimming per cow was included in the study; therefore, the majority of cows trimmed were in the VLS 3 or 4 groups. Only 1.6\% $(n=428)$ of cows detected lame by VLS had a score of 5 . Septic 
arthritis, which can develop from complications of common digit diseases and can result in significant economic losses (Bicalho et al., 2006), was not diagnosed. This suggests that most lameness conditions take longer than a week to progress to this stage, and early intervention can reduce economic losses (Espejo et al., 2006).

Visual detection of lameness was more accurate for hind feet compared with front feet. Prevalence of lameness was higher in hind feet independently of the diagnosing technique. The increased accuracy of VLS might be due to an increased probability of finding problems on the hind legs. It should be noted that hoof overgrowth was not considered a PL and upper limb lameness could account for cows with visual gait abnormalities not having a PL on hoof trimming. As expected, a lower threshold for lameness in LVLS3 resulted in a higher association with a detected PL; the Spearman correlation coefficient for LVLS3 was 0.48 when compared with LVLS4, which had a Spearman correlation coefficient of 0.41 .

For study 2, interobserver agreement among the 3 observers was moderate; the kappa coefficient ranged from 0.45 to 0.48 . O'Callaghan et al. (2003), using percentage agreements, reported interobserver agreement on a 5-point VLS scale of $37 \%$; however, the near agreement defined by the difference of one score was $81 \%$. Engel et al. (2003), using a 9-point classification of gait assessment, found 47\% agreement among 9 observers and an expert after training, which could be improved to $80 \%$, if a difference of one class was allowed. Herein, the ROC analysis was performed for each veterinarian that performed VLS separately, and the areas under the curves did not differ significantly among the veterinarians.

In study 2, a ROC curve analysis for VLS and PL was performed, and the optimal sensitivity-specificity relationship was determined when a cutoff point of VLS $\geq 3$ was used to detect a PL. An ROC curve analysis was performed for the SLS and a PL, and the optimal sensitivity-specificity relationship was determined when a cutoff point of 33 was used to detect a PL. The sensitivities to detect a PL were 67.5 and $33.3 \%$, whereas the specificities were 84.6 and $89.5 \%$, respectively, for VLS and SLS at the optimal cutoff point. The manufacturer's recommended cutoff point for Stepmetrix was 39 , and at this cutoff, the sensitivity was $22.2 \%$ and the specificity was $93.8 \%$. When the ROC curve for VLS was compared with that for SLS to detect a PL, the area under the curve for VLS was significantly larger than that of SLS. Visual locomotion scoring was performed by trained veterinarians in this study and appears to perform better than SLS for detecting lameness. The application of ground reaction force measurements to detect lameness is promising, but may require further development to outperform VLS and become more attractive to dairy producers.

\section{REFERENCES}

Bicalho, R. C., S. H. Cheong, L. D. Warnick, D. V. Nydam, and C. L. Guard. 2006. The effect of digit amputation or arthrodesis surgery on culling and milk production in Holstein dairy cows. J. Dairy Sci. 89:2596-2602.

Clarkson, M. J., D. Y. Downham, W. B. Faull, J. W. Hughes, F. J. Manson, J. B. Merritt, R. D. Murray, W. B. Russell, J. E. Sutherst, and W. R. Ward. 1996. Incidence and prevalence of lameness in dairy cattle. Vet. Rec. 138:563-567.

Engel, B., G. Bruin, G. Andre, and W. Buist. 2003. Assessment of observer performance in a subjective scoring system: Visual classification of the gait of cows. J. Agric. Sci. 140:317-333.

Espejo, L. A., M. I. Endres, and J. A. Salfer. 2006. Prevalence of lameness in high-producing Holstein cows housed in freestall barns in Minnesota. J. Dairy Sci. 89:3052-3058.

Fjeldaas, T., Åc. M. Sogstad, and O. Østerås. 2006. Claw trimming routines in relation to claw lesions, claw shape and lameness in Norwegian dairy herds housed in tie stalls and free stalls. Prev. Vet. Med. 73:255-271.

Flower, F. C., D. J. Sanderson, and D. M. Weary. 2005. Hoof pathologies influence kinematic measures of dairy cow gait. J. Dairy Sci. 88:3166-3173.

Green, L. E., V. J. Hedges, Y. H. Schukken, R. W. Blowey, and A. J. Packington. 2002. The impact of clinical lameness on milk yield of dairy cows. J. Dairy Sci. 85:2250-2256.

Murray, R. D., D. Y. Downham, M. J. Clarkson, W. B. Faull, J. W. Hughes, F. J. Manson, J. B. Merritt, W. B. Russell, J. E. Sutherst, and W. R. Ward. 1996. Epidemiology of lameness in dairy cattle: Description and analysis of digit lesions. Vet. Rec. 138:586-591.

Neveux, S., D. M. Weary, J. Rushen, M. A. G. von Keyserlingk, and A. M. de Passillé. 2006. Hoof discomfort changes how dairy cattle distribute their body weight. J. Dairy Sci. 89:2503-2509.

O'Callaghan, K. A., P. J. Cripps, D. Y. Downham, and R. D. Murray. 2003. Subjective and objective assessment of pain and discomfort due to lameness in dairy cattle. Anim. Welf. 12:605-610.

Rajkondawar, P. G., M. Liu, R. M. Dyer, N. K. Neerchal, U. Tasch, A. M. Lefcourt, B. Erez, and M. A. Varner. 2006. Comparison of model to detect lame cows based on gait and lesion scores, and limb movement variables. J. Dairy Sci. 89:4267-4275.

Sprecher, D. J., D. E. Hostetler, and J. B. Kaneene. 1997. A lameness scoring system that uses posture and gait to predict dairy cattle reproductive performance. Theriogenology 47:1179-1187.

Wells, S. J., A. M. Trent, W. E. Marsh, and R. A. Robinson. 1993. Prevalence and severity of lameness in lactating dairy cows in a sample of Minnesota and Wisconsin herds. J. Am. Vet. Med. Assoc. 202:78-82.

Whay, H. R., D. C. J. Main, L. E. Green, and A. J. F. Webster. 2003. Assessment of the welfare of dairy cattle using animal-based measurements: Direct observations and investigation of farm records. Vet. Rec. 153:197-202. 